\title{
Studying structure formation and evolution with strong-lensing galaxy groups
}

\author{
Gaël Foëx ${ }^{1}$, Veronica Motta ${ }^{1}$, Marceau Limousin ${ }^{2}$, Tomas Verdugo ${ }^{3}$ \\ and Fabio Gastaldello ${ }^{4}$ \\ ${ }^{1}$ Instituto de Física y Astronomía, Universidad de Valparaíso, \\ Avda. Gran Bretaña 1111, Valparaíso, Chile \\ email: gael.foex@uv.cl \\ ${ }^{2}$ Aix Marseille Université, CNRS, Laboratoire d'Astrophysique de Marseille UMR 7326, \\ 13388, Marseille, France \\ ${ }^{3}$ Centro de Investigaciones de Astronomía, \\ AP 264, Mérida 5101-A, Venezuela \\ ${ }^{4}$ INAF - IASF Milano, \\ via E. Bassini 15, I-20133 Milano, Italy
}

\begin{abstract}
We present the analysis of a sample of strong-lensing galaxy group candidates. Our main findings are: confirmation of group-scale systems, complex light distributions, presence of large-scale structures in their surroundings, and evidence of a strong-lensing bias in the massconcentration relation. We also report the detection of the first 'Bullet group'.
\end{abstract}

Keywords. Dark matter, gravitational lensing, galaxy groups.

\section{The SARCS sample}

The Strong Lensing Legacy Survey (SL2S, Cabanac et al. 2007) is a semi-automated search of strong-lensing systems on the full Canada-France-Hawaii Telescope Legacy Survey (CHFTLS). Using the ARCFINDER algorithm, More et al. (2012) compiled the SL2S-ARCS sample (SARCS) made of group- and cluster-scale lens candidates. Basically, ARCFINDER searches for elongated and contiguous features of pixels above a given intensity threshold, and tags the most promising features as arc candidates according to their width, length, area, and curvature. Roughly 1000 candidates $/ \mathrm{deg}^{2}$ were found, which were then inspected visually to reduce the sample to 413 systems $\left(\sim 2.75\right.$ candidates $\left./ \mathrm{deg}^{2}\right)$. The most promising candidates with an arc radius $\mathrm{R}_{\mathrm{A}} \gtrsim 2$ " were kept, leading to a total of 127 objects (More at al., 2012). The sample spans a redshift range $z \in[0.2-1.2]$ and peaks at $\mathrm{z} \sim 0.5$. The SARCS distribution of image separation is located between the galaxy-scale SLACS sample and the massive cluster MACS sample, thus corresponding mostly to groups and poor clusters of galaxies.

\section{Weak-lensing and optical analyses}

For each SARCS candidate, we the fitted the measured shear profile by the singular isothermal sphere (SIS) mass model to estimate the velocity dispersion $\sigma_{v}$. We also constructed luminosity maps using the bright galaxies populating the red sequence. We combined these two analyses to build a sample of 80 most secure lens candidates, characterized by a positive weak-lensing detection $\left(\sigma_{v}-\sigma>0\right)$ and a clear light over-density associated to the strong-lensing feature (Foëx et al. 2013). With this reduced sample, we investigated the optical scaling relations of strong-lensing galaxy groups. Despite a 
large scatter (up to $35 \%$ ), we found correlations between the SIS $\sigma_{v}$ and the optical richness and luminosities. We combined the SARCS sample with a sample of massive galaxy clusters (Foëx et al., 2012) to derive scaling laws consistent with the expectations of the hierarchical model of structure formation and evolution.

The morphological study of the luminosity maps revealed that a significant fraction of groups present a complex light distribution: $\sim 42 \%$ with highly-elongated luminosity contours, $\sim 16 \%$ with a multimodal structure (Foëx et al., 2013). These results suggest that galaxy groups are dynamically-young objects, a picture consistent with a temporary stage towards the formation of more massive clusters. We also inspected the groups' luminosity map at larger scales. We found 10 systems with crowded environments made of several light over-densities not randomly distributed, suggesting the presence of largescale filamentary structures (Foëx et al., in prep.).

In a second paper (Foëx et al., 2014), we performed a stacked weak-lensing analysis to constrain the $c(M)$ mass-concentration relation of strong lenses. We found an average concentration $c_{200}=8.6 \pm 1.8$ for an average $M_{200}=(0.73 \pm 0.1) \times 10^{14} \mathrm{M}_{\odot}$, a concentration in disagreement at the $3 \sigma$ level with the predictions from numerical simulations (Duffy et al., 2008). We combined our composite strong lenses with massive strong-lensing galaxy clusters to derive the $c(M)$ over nearly two decades in mass. We found a relation much steeper than expected, resulting from projections effects of highly-elongated haloes with a major axis close to the line of sight.

\section{The 'Bullet Group'}

A deeper investigation of the group SL2S J08544-0121 revealed a separation of $124 \pm 20$ $\mathrm{kpc}$ between the X-ray emission peak and the mass centers of this bi-modal system (Gastaldello et al. 2014). Such a separation between the collisional gas and the collisionless galaxies and dark matter is characteristic of merging systems in the plan of the sky, as the so-called 'Bullet Cluster'. The estimated mass of the system is $M_{200}=$ $(2.4 \pm 0.6) \times 10^{14} \mathrm{M}_{\odot}$ from a M-T scaling relation, and $M_{200}=(2.2 \pm 0.5) \times 10^{14} \mathrm{M}_{\odot}$ from the weak-lensing analysis, which makes it the lowest mass bullet-like object found to date. We used this 'Bullet Group' to derive an upper limit on the dark matter selfinteraction cross-section of $10 \mathrm{~cm}^{2} \mathrm{~g}^{-1}$. We showed in a parallel study based on numerical simulations (Fernandez-Trincado et al. 2014) that bullet groups are more numerous than massive bullet clusters. Therefore, with this first detection of a low-mass bullet-like system, we prove the possibility of using galaxy groups to perform a statistical study of the dark matter cross-section.

G.F. acknowledges funds from FONDECYT grant \#3120160. V.M. acknowledges funds from FONDECYT grant \#1120741. GF, VM, ML acknowledge funds from ECOS-CONICYT C12U02.

\section{References}

Cabanac, R., et al. 2007, A\&A, 461, 813

Duffy, A. R., et al. 2008, MNRAS, 390, 64

Gastaldello, F., et al. 2014, MNRAS, 442, 76

Fernandez-Trincado, J. G., et al. 2014, ApJ, 787, 34

Foëx, G., et al. 2012, A\& $A, 546,106$

Foëx, G., et al. 2013, A\&sA, 559, 105

Foëx, G., et al. 2014, ArXiv, 1409.5905

More, A., et al. 2012, ApJ, 749, 38 